\title{
INFLUÊNCIA DO ALUMÍNIO NO CRESCIMENTO E NA ACUMULAÇÃO DE NUTRIENTES EM MUDAS DE GOIABEIRA ${ }^{(1)}$
}

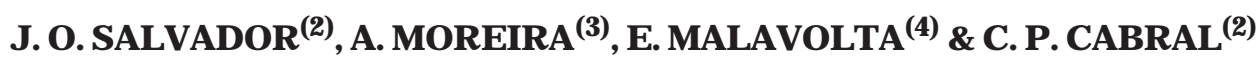

\begin{abstract}
RESUMO
Durante 110 dias, realizou-se um experimento em solução nutritiva, para avaliar os efeitos do alumínio nas concentrações de 0, 5, 10, 15, 20 e $25 \mathrm{mg} \mathrm{L}^{-1}$, em pH 4,0, sobre o crescimento de mudas de goiabeira, absorção e acumulação de macronutrientes e de $\mathrm{Cu}, \mathrm{Fe}, \mathrm{Mn}, \mathrm{Zn}$ e Al. Os sintomas visuais de fitotoxidez, associados às doses 20 e $25 \mathrm{mg} \mathrm{L}^{-1}$ de $\mathrm{Al}$, foram caracterizados pela menor altura das plantas, ausência de ramificação, menor diâmetro do caule e raízes mais escuras; as folhas apresentaram dimensões menores e pecíolos avermelhados. Em doses relativamente baixas, o alumínio promoveu resposta positiva de crescimento, porém, em doses altas, os teores de macronutrientes e de Fe, Mn e Zn nas folhas de diagnose (30 e $4^{\circ}$ pares a partir do ápice), bem como o conteúdo mineral nas diversas partes da planta, foram influenciados pelas doses de alumínio. A fitotoxidez afetou, mais acentuadamente, os teores de P, Ca, Mg, Mn e Zn. Cerca de $95 \%$ do Al absorvido concentrou-se nas raízes.
\end{abstract}

Termos de indexação: Psidium guajava, concentração de nutrientes, fitotoxidez de alumínio, fruticultura.

\section{SUMMARY: ALUMINUM INFLUENCE ON GROWTH AND NUTRIENT ACCUMULATION BY YOUNG GUAVA PLANTS}

Young guava plants weregrown in a nutrient solution in the presence of $0,5,10,15,20$ and $25 \mathrm{mg} \mathrm{L}^{-1}$ al uminum, with $\mathrm{pH}$ being kept at 4.0. After 110 days, theplants wereharvested and their content of macronutrients, $\mathrm{Cu}, \mathrm{Fe}, \mathrm{Mn}, \mathrm{Zn}$ and $\mathrm{Al}$ weredetermined. Visual toxicity symptoms, developed under 20 and $25 \mathrm{mg} \mathrm{L}^{-1}$ aluminum, wereas foll ows: reduction in height,

\footnotetext{
(1) Recebido para publicação em novembro de 1999 e aprovado em maio de 2000.

(2) Biólogo do Centro de Energia Nuclear na Agricultura - CENA. Av. Centenário 303. Caixa Postal 96, CEP 13400-970 Piracicaba (SP). E-mail: salvador@cena.usp.br; cpcabral@cena.usp.br

(3) Engenheiro-Agrônomo, Embrapa Amazônia Ocidental. Caixa Postal 319, CE P 69011-970, Manaus (AM). Bolsista do DCR/CN Pq. E-mail: adonis@cpaa.embrapa.br

(4) Engenheiro-Agrônomo, Pesquisador do CENA/USP. Bolsista do CNPq. E-mail: mala@cena.usp.br
} 


\begin{abstract}
absence of branching, decreasein diameter of stem dark roots and smaller leaves presenting reddish petiole. Dry matter was also reduced. The macronutrient, $\mathrm{Fe}, \mathrm{Mn}$ and $\mathrm{Zn}$ concentrations in the leaves sampled (third and fourth pairs from the top) were affected by Al rates. The same did occur with the content of el ements in the various parts of the plant. Aluminum toxicity affected particularly the content of $\mathrm{P}, \mathrm{Ca}, \mathrm{Mg}, \mathrm{Fe}$, and $\mathrm{Mn}$ with approxi matel $y 95 \%$ of theAl taken up confined to theroot system. Lower rates of aluminum promoted growth.
\end{abstract}

Index terms: Psidium guajava, nutrient concentration, aluminum toxicity, fruit growing.

\section{INTRODUÇÃO}

Dentre as espécies frutíferas de mirtáceas cultivadas, a goiabeira (Psidium guajava L.) éa mais conhecida e importante, participando de grandes projetos comerciais de fruticultura. No Brasil, terceiro maior produtor mundial de goiaba, ela é cultivada de norte a sul (Gonzaga Neto \& Soares, 1995), passando a receber maior atenção, somente na última década com a introdução de variedades selecionadas, com vistas em atender às exigências das indústrias.

A espontaneidade no desenvolvimento nos mais diversos tipos de solos e ambientes dá à cultura a conotação de planta rústica ou de relativa tolerância a condições adversas. Todavia, pesquisas realizadas por Martinez J r. \& Pereira (1986), Natale et al. (1996) eSalvador et al. (1998; 1999a,b) demonstram quea goiabeira revela al ta demanda por nutrientes, o que, naturalmente, requer maior atenção no acompanhamento técnico para a elaboração de programas de correção da acidez e de suprimento de nutrientes.

A baixa resposta das plantas às adubações pode ser atribuída, em parte, ao excesso dealumínio que, em solo com elevada acidez, pode tornar-se tóxico, causando graves anomalias ao sistema radicular, modificando os padrões de absor ção e o metabol ismo de nutrientes. Os efeitos nocivos do alumínio para al gumas espécies vegetais são mencionados por Clarkson (1969), F oy et al. (1978), Furlani (1989) e F oy (1992), porém não há registros detrabal hos que abordam os reflexos negativos do excesso desse el emento na cultura da goiabeira. Sabe-se que sua ocorrência é bastante representativa nos solos brasileiros, principal mente naqueles sob vegetação de Cerrado que, além de ácidos, são carentes em $P$, Ca e Mg (Lopes, 1984).

Os efeitos fitotóxi cos causados pel o al umínio são al tamentedependentes de pH, concentrações de sais, compostos orgânicos, temperatura e espécie vegetal, fatores que podem ser alterados de acordo com a natureza da composi ção química do substrato ou da espécie estudada (Camargo \& Furlani, 1989).

O presente trabal hoteve por finalidadeavaliar o efeito de doses de alumínio, em solução nutritiva, sobre o crescimento, disponibilidade, absorção, interação e redistribuição de N, P, K, Ca, Mg, S, Cu, $\mathrm{Fe}, \mathrm{Mn}, \mathrm{Zn}$ e Al em mudas de goiabeira.

\section{MATERIAL E MÉTODOS}

O presente trabalho foi realizado em casa de vegetação do Centro de Energia Nuclear na Agricultura - CENA/USP (Piracicaba - SP), situado nas coordenadas $22^{\circ} 42^{\prime} 30^{\prime \prime}$ de LS e $47^{\circ} 38^{\prime} 00^{\prime \prime}$ de LW.

As plantas de goiabeira com sete pares de fol has, obti das de sementes deuma planta nativa com frutos de polpa vermelha, foram transferidas para recipientes de plástico com dois litros de solução nutritiva - 1/5 da solução no 1 de H oagland \& Arnon (1950), correspondendo, em mg L-1: 42 de $\mathrm{N}-\mathrm{NO}_{3}$; 6,2 de $\mathrm{P} ; 46,8$ de $\mathrm{K} ; 40$ de $\mathrm{Ca} ; 9,6$ de $\mathrm{Mg} ; 12,8$ de $\mathrm{S}$ $\mathrm{SO}_{4} ; 0,5$ de $\mathrm{B} ; 0,02$ de $\mathrm{Cu} ; 0,5$ de $\mathrm{Mn} ; 0,01$ de $\mathrm{Mo}$; 0,05 de $\mathrm{Zn}$ e 5,0 de Fe. O Fe foi fornecido na forma de Fe-EDTA, de acordo com Malavolta (1976). Os tratamentos constituíram-se de doses de Al; $0,5,10$, 15,20 e $25 \mathrm{mg} \mathrm{L}^{-1}$, adicionados como $\mathrm{AlCl}_{3}$. O experimento foi distribuído em delineamento experimental inteiramente casualizado, com três repetições, e uma planta por vaso. $\mathrm{O}$ pH da solução era ajustado para $4 \pm 0,2$, três vezes ao dia, com a solução de $\mathrm{HCl}$ 0,1 mol L-1 ou $\mathrm{NaOH} 0,1 \mathrm{~mol} \mathrm{~L}^{-1}$, visando minimizar a precipitação do alumínio. O volume de cada vaso foi mantido constante com adições diárias de água destilada e desionizada. As sol uções foram renovadas semanal mente para manter as concentrações dealumínioe de nutrientes próximas aos val ores preestabel ecidos. Após a adição do Al, os valores de $\mathrm{pH}$ verificados nas diversas soluções foram: dose de $0 \mathrm{mg} \mathrm{L}^{-1}$, pH 6,0; dose de $5 \mathrm{mg} \mathrm{L}^{-1}$, pH 5,9; dose de $10 \mathrm{mg} \mathrm{L}^{-1}$, pH 3,7; dose de $15 \mathrm{mg} \mathrm{L}^{-1}, \mathrm{pH} \mathrm{3,5}$; dose de $20 \mathrm{mg} \mathrm{L}^{-1}$, pH 3,5; dose de $25 \mathrm{mg} \mathrm{L}^{-1}, \mathrm{pH} 3,5$. O experimento teve duração de 110 dias.

Por meio de avaliação biométrica, foram estimados: aos 30 dias, a altura da planta, o comprimento transversal eolongitudinal da 3 a fol ha; aos 90 dias, a área foliar desse tipo de folha pelo método da fotocópia e, aos 110 dias, a produção da matéria seca decaules, fol has e raízes e a medida do 
diâmetro do caule. Também foram avaliados as concentrações e os acúmul os de macronutrientes $(\mathrm{N}$, $\mathrm{P}, \mathrm{K}, \mathrm{Ca}, \mathrm{MgeS}$ ) emicronutrientes (Cu, Fe, Mn eZn) e de Al no conjunto de dois pares de fol has maduras mais jovem do eixo principal, correspondendo ao $3^{\circ}$ e 4ํpar, aos 60 dias; concentrações e quantidades de nutrientes e de alumínio nas folhas, caules e raízes, aos 110 dias.

Para a descontaminação externa do alumínio, as raízes foram submetidas a uma lavagem rápida em solução de EDTA - ácido a 0,02 mol L-1, seguida da lavagem em $\mathrm{HCl}$ 0,1 mol L-1 e, por fim, em água destilada; fol has e caules foram lavados em água corrente e, posteriormente, em água destilada. O material col etado foi seco em estufa, pesado, moído e digerido para a obtenção dos extratos. Os teores de $\mathrm{P}$ foram determinados pelo método da colorimetria de molibdovanadato; os de K, por fotometria dechama; os de $\mathrm{Ca}, \mathrm{Mg}, \mathrm{Cu}, \mathrm{Fe}, \mathrm{Mn}$ e Zn, por espectrometria de absorção atômica; os deS, por turbidimetria do $\mathrm{BaSO}_{4}$; e os de $\mathrm{N}$, pelo método semimicro Kjeldahl. O Al foi determinado pelo método aluminon, para as fol has de diagnose, e por espectrometria de emissão atômica em plasma de argônio (ICP-AES), para as partes vegetais. Todos os métodos analíticos utilizados estão descritos em Malavolta et al. (1997).

Os dados de crescimento e acumulação foram submetidos à análise de variância, Teste $F(5 \%)$ e anál ise de regressão, de acordo com Pimentel-Gomes (1990).

\section{RESULTADOS E DISCUSSÃO}

Observou-se que, após os ajustes de $\mathrm{pH}$, as soluções que continham alumínio em maiores doses permaneciam com os índices bastante estáveis. De acordo com Foy (1984) e Cambraia (1989), uma das características de algumas espécies é a habilidade de elevar o pH do meio no qual se desenvolvem, provocando a preci pitação do el emento pela absorção diferencial de cátions e ânions, reduzindo sua concentração em soluç̧ão, ficando menos expostas aos efeitos nocivos do el emento. Diante dessas afirmações, presume-se quea goiabeira não pertença ao grupo de plantas tolerantes ao excesso de Al, por não conseguir elevar os níveis de pH, mantendo-o numa forma inócua. Deve-se acrescentar, no entanto, que o comportamento diferencial entre plantas para tolerância pode variar de acordo com as espécies e dentro de uma mesma espécie (Furlani, 1983).

\section{Sintomas visuais de fitotoxidez}

As doses de Al acima de $15 \mathrm{mg} \mathrm{L-1}$ ocasionaram menor altura das plantas, ausência de ramificações, fol has com bordos apresentando certas ondulações, assemel hando-se, de certa forma, com uma das características de deficiência de fósforo, descrita por Salvador et al. (1998). O pecíolo apresentou-se de tonalidade avermelhada e as raízes de coloração mais escura. Segundo Furlani (1989), os sintomas visuais da fitotoxidez de alumínio na parte aérea também se assemel ham aos de deficiência de fósforo e o suprimento deste ocasiona um efeito protetivo contra a injúria do Al.

Muitos pesquisadores acreditam que os efeitos da fitotoxidez de alumínio podem ser atribuídos diretamente a uma deficiência de $\mathrm{P}$ induzida pelo Al (Foy, 1992). Para Oliveira (1979), esse pressuposto efeito sobre o metabolismo do fósforo pode estar ligado principal mente à sua retenção radicular e à redução de sua translocação para a parte aérea.

\section{Avaliação biométrica}

Os efeitos das doses de alumínio para altura da planta, comprimento elargura da fol ha, área foliar ediâmetro do caule (Figura 1), obtidos notratamento sem adição de Al (testemunha), foram significativamente menores dos que os das plantas presentes em solução de 5 e $10 \mathrm{mg} \mathrm{L}^{-1}$ de Al, porém superiores àqueles encontrados nas plantas submetidas às doses de 20 e 25 mg L-1. Para o peso de matéria seca (MS) de caule, fol has eraiz (Quadro 1), os resultados foram similares àqueles observados em outras variáveis mensuradas. O peso da MS das fol has foi superior na dose de $5 \mathrm{mg} \mathrm{L-1}$, com redução significativa a partir de $15 \mathrm{mg} \mathrm{L-1;}$; nas duas últimas doses de alumínio, o peso médio de MS desse segmento atingiu a metade do obtido pelo tratamento-testemunha. As doses de 5 e $10 \mathrm{mg} \mathrm{L}-1$ proporcionaram maiores valores para o peso da MS do caule, enquanto as doses de 20 e $25 \mathrm{mg} \mathrm{L}^{-1}$ mostraram uma redução aproximada de $40 \%$ em relação à testemunha. Para a raiz, o maior peso de MS foi obtido nas plantas desenvolvidas na dose de $5 \mathrm{mg} \mathrm{L}^{-1}$ de Al, valor que representa, praticamente, o dobro quando comparado com qualquer tratamento. Quantoà MS total (Quadro 1), percebeuse que, em concentrações rel ativamente baixas, o Al promoveu resposta positiva de crescimento $(0 \mathrm{a}$ $15 \mathrm{mg} \mathrm{L}-1)$. As ações fitotóxicas do alumínio relacionaram-se mais com a parte aérea, o que, via de regra, contraria certas linhas de evidência de que o sistema radicular é mais sensível ao alumínio do que os órgãos da parte aérea.

Os efeitos estimulantes (Figura 1 e Quadro 1) também foram encontrados por Mullette(1975), para Eucalyptus; Fageria \& Zimmermann (1979), para arroz; Oliveira \& Malavolta (1982), para feijão, e Bueno(1997), para o cupuaçu. Tais efeitos, segundo Foy (1974) e Asher (1991), ainda não são bem conhecidos. Mesmo havendo especulacões sobre a sua essencialidade, a ausência de métodos confiáveis e sensíveis para a sua determinação em amostra biológica faz com que a pressuposta essencialidade permaneça ainda no campo das hipóteses (Asher, 
Quadro 1. Produção de matéria seca das folhas, caule, raízes e total, aos $\mathbf{1 1 0}$ dias, de mudas de goiabeira de acordo com as doses de alumínio

\begin{tabular}{cllcc}
\hline \multirow{2}{*}{ Dose de Al } & \multirow{2}{*}{ Folha } & Caule & Raízes & Total \\
\hline mg L-1 & \multicolumn{4}{c}{ g/planta } \\
\cline { 2 - 5 } 0 & 25,30 & 19,26 & 11,35 & 55,92 \\
5 & 33,81 & 31,08 & 20,77 & 85,65 \\
10 & 26,98 & 28,09 & 12,83 & 67,90 \\
15 & 12,01 & 19,46 & 12,54 & 44,02 \\
20 & 11,79 & 11,91 & 9,63 & 33,34 \\
25 & 10,84 & 11,29 & 10,56 & 32,69 \\
Teste F & $20,412^{* *}$ & $16,240 * *$ & $5,514 * *$ & $14,011^{* *}$ \\
C.V. (\%) & 16,522 & 17,297 & 22,871 & 17,468 \\
\hline
\end{tabular}

** significativo a $1 \%$.
1991). Dentre as dificuldades para estabelecer um conhecimento pleno sobre os efeitos fisiológicos e bioquímicos do Al, destacam-se: a complexidade no entendimento da físi co-química do Al; a ausência de radioisótopo adequado; os problemas de contaminação na análise química, decorrentes de sua natureza ubíqua (Furlani, 1989).

De qualquer forma, algumas possíveis causas dessa ação benéfica são discutidas por F oy (1974) e Marschner (1995), figurando, entre elas, a correção ou prevenção da deficiência de Fe; a liberação de cátions adsorvidos em sítios metabolicamenteinativos dentro da planta; o bloqueio de sítios, na parede celular, carregados negativamente, promovendo menor absorção de P; a prevenção da toxidez de Cu e Mn. A possibilidade de propiciar maior equilíbrio de nutrientes no meio de crescimentoéuma hipótese defendida por Fageria \& Zimmermann (1979).
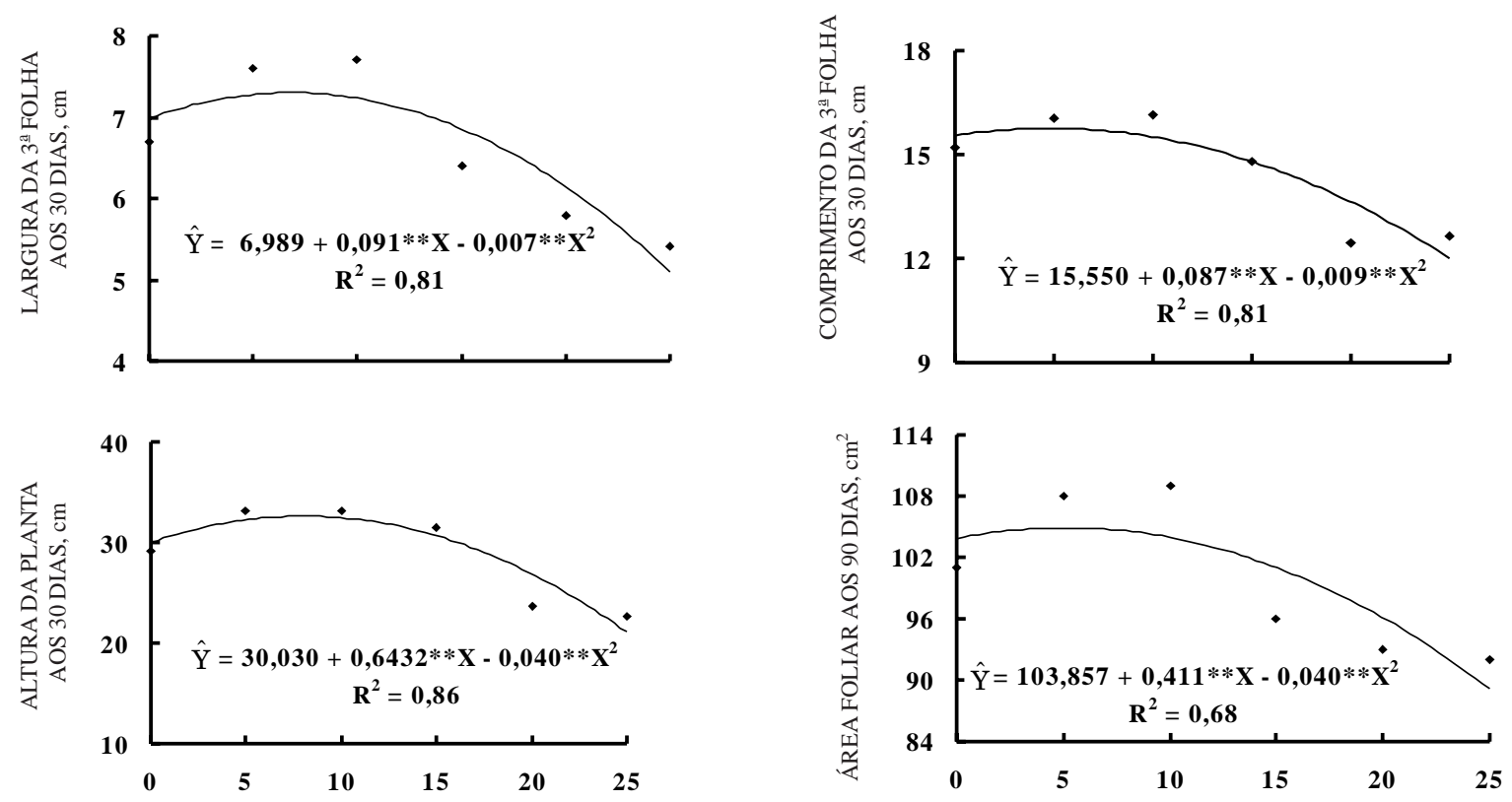

DOSES DE Al, mg L ${ }^{-1}$

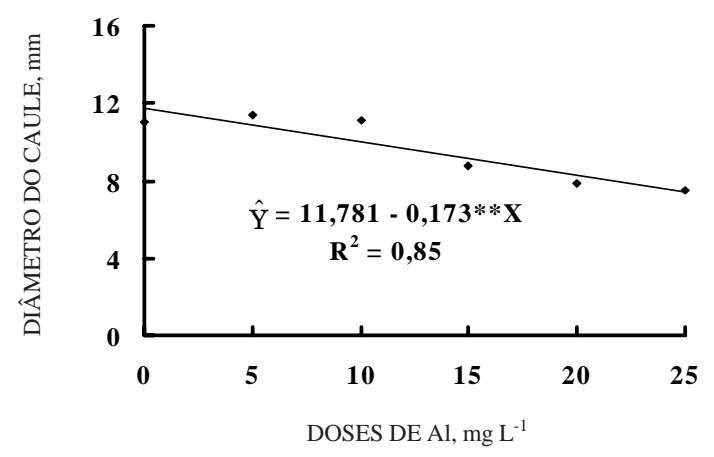

Figura 1. Biometria de largura e comprimento da $3^{a}$ folha, altura da planta, aos 30 dias; área foliar da $3^{a}$ folha, aos 90 dias e, diâmetro do caule, aos 110 dias de mudas de goiabeira (* significativo a 1\%). 


\section{E feitos do alumínio nos teores de nutrientes e alumínio nas folhas usadas para diagnóstico}

Dentre os macronutrientes, o $\mathrm{N}$ e K foram menos afetados em seus teores pelas doses de Al do que $\mathrm{P}$, $\mathrm{Ca}, \mathrm{MgeS}$, quetiveram um decréscimo significativo em seus teores, à medida que seaumentou a dose de alumínio, atingindo reduzidas concentrações nos dois maiores níveis do elemento, perfazendo, praticamente, a metade das contidas na testemunha (Quadro 2). Conforme Foy (1974) e Furlani (1989), essa redução podeestar relacionada com a interferência do excesso do alumínio nas reações enzimáticas ena disposição depolissacarídeos nas paredes celulares, prejudicando, com isso, a absorção, o transporte e o uso de vários nutrientes, entre eles $\mathrm{P}, \mathrm{Ca}$ e Mg. Com referência aos micronutrientes, exceto o $\mathrm{Cu}$, os teores foliares deFe, $\mathrm{Mn}$ e Zn apresentaram alterações relacionadas com as doses de alumínio (Quadro 2). Para o Fe e $\mathrm{Mn}$, a maior concentração de Al diminuiu significativamente os seus teores, enquanto a de $\mathrm{Zn}$ aumentou nas três primeiras adições. Resultados semel hantes também foram relatados por Foy (1984) e Asher (1991).

Os teores de al umínio aumentaram ( $p \leq 0,01)$ com o incremento de sua concentração na sol ução (Quadro 3). O teor máximo obtido dispôs-se sob uma faixa correspondente à das plantas não acumuladoras de Al - <400 $\mathrm{mg} \mathrm{kg}^{-1}$ (F oy, 1984). Ainda, de acordo com o autor, plantas com teores mai ores de $1.000 \mathrm{mg} \mathrm{kg}^{-1}$ de alumínio nas fol has são consideradas acumuladoras, porém, por meio de mecanismos de proteção interna, defendem-se de seus efeitos maléficos.

Quadro 2. Concentração de nutrientes e de Al, aos 60 dias, nas folhas de diagnose ( $3^{\circ}$ e $4^{\circ}$ par), de mudas de goiabei ra de acordo com as doses de Al

\begin{tabular}{|c|c|c|c|}
\hline Nutriente & & E quação & $\mathbf{R}^{2}$ \\
\hline \multicolumn{4}{|c|}{$\mathrm{g} \mathrm{kg}^{-1}$} \\
\hline $\mathrm{N}$ & $\hat{\mathrm{Y}}$ & $=28,208-0,282 * * X$ & 0,64 \\
\hline$P$ & $\hat{\mathrm{Y}}$ & $=2,089-0,050 * * X$ & 0,96 \\
\hline K & $\hat{\mathrm{Y}}$ & $=33,064-0,773 X^{(0,21)}+0,028 * X^{2}$ & 0,38 \\
\hline $\mathrm{Ca}$ & $\hat{\mathrm{Y}}$ & $=10,383-0,251 * * x$ & 0,88 \\
\hline $\mathrm{Mg}$ & $\hat{\mathrm{Y}}$ & $=2,877-0,050 * * x$ & 0,83 \\
\hline s & $\hat{\mathrm{Y}}$ & $=3,992-0,080 * * x$ & 0,97 \\
\hline \multicolumn{4}{|c|}{$\mathbf{m g ~ \mathbf { ~ k g } ^ { - 1 }}$} \\
\hline $\mathrm{Cu}$ & $\hat{\mathrm{Y}}$ & $=7,460-0,059^{(0,13)} \times$ & 0,25 \\
\hline $\mathrm{Fe}$ & $\hat{\mathrm{Y}}$ & $=254,937-3,804 * * x$ & 0,63 \\
\hline $\mathrm{Mn}$ & $\hat{\mathrm{Y}}$ & $=139,1269-1,859 * x$ & 0,43 \\
\hline $\mathrm{Zn}$ & $\hat{\mathrm{Y}}$ & $=22,917+1,962 * x-0,094 * * x^{2}$ & 0,79 \\
\hline Al & $\hat{\mathrm{Y}}$ & $=223,136+1,889 * * x$ & 0,87 \\
\hline
\end{tabular}

* e**significativos a 5 e $1 \%$, respectivamente. Os valores entre parênteses representam a significância dos coeficientes de regressão não significativas nem a 5\%.

\section{Efeitos do alumínio nos teores de nutrientes e alumínio nas folhas, caule e raízes}

As doses de Al afetaram significativamente a composição mineral das três partes vegetais (Quadro 3), à exceção da raiz, em termos de N e S. De modo geral, as alterações de maior relevância ocorreram para P, Ca eMg. No caso do Ca, seus teores nas doses de 0,0 mg e 5,0 mg L-1 de alumínio foram parecidos, a partir das quais ocorreram decréscimos nas fol has e caule, atingindo proporções maiores, de acordo com o aumento nas adi ções do metal. Para o $\mathrm{Mg}$, os teores no caule atingiram reduções de, aproximadamente, $50 \%$ em todos os tratamentos em que o Al foi adicionado; nas raízes, essas reduções ocorreram, mais acentuadamente, nas duas últimas doses.

Deacordo com Faquin \& Vale (1991) e Malavolta et al. (1997), o alumínio, quando na forma solúvel, além decausar fitotoxidez nas plantas, compete com outros cátions, principalmente $\mathrm{Ca}$ e $\mathrm{Mg}$, pelos mesmos sítios de absorção no complexo de troca. Quanto ao fósforo, houve diminuições nos teores do caule e das fol has e aumentos nas raízes. Maiores concentrações de $\mathrm{P}$ nas raízes podem estar mais relacionadas com o transportee com a redistribuição do que propriamente com sua absorção. Conforme Oliveira (1979), é provável que parte do alumínio contido nos tecidos radiculares tenha precipitado parte do fósforo absorvido (AI-P), reduzindo, desta maneira, sua translocação para a parte aérea. Braccini et al. (1995), estudando o comportamento do cafeeiro quanto à tolerância ao Al, associaram o grau de tol erância ligado à menor preci pitação de $\mathrm{P}$ nas raízes e à menor redução de transl ocação para a parte aérea.

Com relação aos micronutrientes (Quadro 3), não foram verificadas diferenças significativas para os teores de ferro nos três órgãos analisados (fol has, caule e raízes). Para o cobre, essa significância foi registrada somente no caule e, para o Zn, na raiz. Os maiores teores deCu e de F eobservados na raiz, em relação aos contidos nas fol has, independeram de tratamento e refletiram a baixa redistribuição desses nutrientes (Malavolta et al., 1997). Para o al umínio, observaram-se acréscimos nos seus teores nas fol has e raízes, dependendo das adições do metal (Quadro 3). Nas fol has, para cada aumento de concentração de Al, ocorreu um incremento de cerca de $40 \mathrm{mg} \mathrm{kg}^{-1}$ de Al; na raiz, as maiores doses de alumínio el evaram seus teores radiculares 60 vezes, em relaçãoà testemunha; nessecaso, ocaulefoi oórgão menos afetado pelas doses de alumínio.

\section{Efeitos do Al no acúmulo de macronutrientes nas folhas, caule e raízes}

De maneira geral, os aumentos no acúmulototal dos nutrientes nas três partes da pl anta relacionaramse com as doses de 0 e $5 \mathrm{mg} \mathrm{L}^{-1}$ e as reduções com as doses de 20 e $25 \mathrm{mg} \mathrm{L}^{-1}$ deAl (Figura 2). Baseando no 
Quadro 3. Equação de regressão e coeficientes de determi nação da composi ção de nutrientes e de Al nas diversas partes da planta de mudas de goiabeira de acordo com as doses de alumínio

\begin{tabular}{|c|c|c|c|}
\hline \multirow{2}{*}{ Elemento } & \multicolumn{3}{|c|}{ Órgão da planta } \\
\hline & Folha & Caule & Raiz \\
\hline & \multicolumn{3}{|c|}{$\mathbf{g ~ k g}^{-1}$} \\
\hline $\mathrm{N}$ & $\begin{aligned} \hat{Y} & =16,565+0,168 * X \\
R^{2} & =0,58\end{aligned}$ & $\begin{aligned} \hat{\mathrm{Y}} & =6,202-0,209 * * \mathrm{X}+0,008^{* *} \mathrm{X}^{2} \\
\mathrm{R}^{2} & =0,72\end{aligned}$ & $\begin{aligned} \hat{Y} & =10,871+0,027^{(0,12) X} \\
R^{2} & =0,05\end{aligned}$ \\
\hline$P$ & $\begin{aligned} \hat{\mathrm{Y}} & =1,543-0,028 * * \mathrm{X} \\
\mathrm{R}^{2} & =0,86\end{aligned}$ & $\begin{aligned} \hat{\mathrm{Y}} & =0,721-0,020 * * \mathrm{X} \\
\mathrm{R}^{2} & =0,84\end{aligned}$ & $\begin{aligned} \hat{Y} & =1,571+0,097 * X-0,004 * X^{2} \\
R^{2} & =0,65\end{aligned}$ \\
\hline K & $\begin{aligned} \hat{\mathrm{Y}} & =21,481+0,375 * \mathrm{X} \\
\mathrm{R}^{2} & =0,68\end{aligned}$ & $\begin{aligned} \hat{\mathrm{Y}} & =8,925-0,157 * * \mathrm{X}+0,011^{* * \mathrm{X}^{2}} \\
\mathrm{R}^{2} & =0,66\end{aligned}$ & $\begin{aligned} \hat{Y} & =17,660+0,288 * X \\
R^{2} & =0,47\end{aligned}$ \\
\hline $\mathrm{Ca}$ & $\begin{aligned} \hat{\mathrm{Y}} & =11,699-0,243 \mathrm{X} \\
\mathrm{R}^{2} & =0,97\end{aligned}$ & $\begin{aligned} \hat{Y} & =6,492-0,062 * * X+0,011 * * X^{2} \\
R^{2} & =0,82\end{aligned}$ & $\begin{aligned} \hat{Y} & =5,465-0,802 * * X \\
R^{2} & =0,82\end{aligned}$ \\
\hline $\mathrm{Mg}$ & $\begin{aligned} \hat{Y} & =2,642-0,035^{* *} \mathrm{X} \\
\mathrm{R}^{2} & =0,89\end{aligned}$ & $\begin{aligned} \hat{Y} & =0,879-0,016 * X \\
R^{2} & =0,50\end{aligned}$ & $\begin{aligned} \hat{Y} & =2,014-0,049 * * X \\
R^{2} & =0,72\end{aligned}$ \\
\hline \multirow[t]{2}{*}{$\mathrm{S}$} & $\begin{aligned} \hat{\mathrm{Y}} & =3,190-0,032 * * \mathrm{X} \\
\mathrm{R}^{2} & =0,91\end{aligned}$ & $\begin{aligned} \hat{Y} & =1,529-0,068 * X+0,002 * X^{2} \\
R^{2} & =0,64\end{aligned}$ & $\begin{aligned} \hat{Y} & =3,336-0,001^{(0,13)} X \\
R^{2} & =0,01\end{aligned}$ \\
\hline & \multicolumn{3}{|c|}{ mg kg-1 } \\
\hline $\mathrm{Cu}$ & $\begin{aligned} \hat{\mathrm{Y}} & =3,170+0,019^{(0,11)} \mathrm{X} \\
\mathrm{R}^{2} & =0,16\end{aligned}$ & $\begin{aligned} \hat{Y} & =4,018-0,142 * X+0,005 * X^{2} \\
R^{2} & =0,59\end{aligned}$ & $\begin{aligned} \hat{Y} & =8,481+0,046^{(0,13)} X \\
R^{2} & =0,15\end{aligned}$ \\
\hline $\mathrm{Fe}$ & $\begin{aligned} \hat{\mathrm{Y}} & =120,571-0,006 * \mathrm{X} \\
\mathrm{R}^{2} & =0,05\end{aligned}$ & $\begin{aligned} \hat{\mathrm{Y}} & =40,190-0,509^{(0,18)} \mathrm{X} \\
\mathrm{R}^{2} & =0,23\end{aligned}$ & $\begin{aligned} \hat{Y} & =554,524+17,416^{(0,03) X}-0,799^{(0,22)} X^{2} \\
R^{2} & =0,05\end{aligned}$ \\
\hline $\mathrm{Mn}$ & $\begin{aligned} \hat{\mathrm{Y}} & =142,016-2,312 * * \mathrm{X} \\
\mathrm{R}^{2} & =0,87\end{aligned}$ & $\begin{aligned} \hat{\mathrm{Y}} & =46,746-0,789 * * \mathrm{X} \\
\mathrm{R}^{2} & =0,71\end{aligned}$ & $\begin{aligned} \hat{Y} & =123,571-2,766 * * X \\
R^{2} & =0,93\end{aligned}$ \\
\hline $\mathrm{Zn}$ & $\begin{aligned} \hat{Y} & =16,063+0,044 * X \\
R^{2} & =0,08\end{aligned}$ & $\begin{aligned} \hat{Y} & =10,111-0,160 * X \\
R^{2} & =0,43\end{aligned}$ & $\begin{aligned} \hat{Y} & =40,302-0,829 * * X \\
R^{2} & =0,96\end{aligned}$ \\
\hline $\mathrm{Al}$ & $\begin{aligned} \hat{\mathrm{Y}} & =41,032+7,922 * * \mathrm{X} \\
\mathrm{R}^{2} & =0,99\end{aligned}$ & $\begin{aligned} \hat{\mathrm{Y}} & =19,964-0,465(0,26) \mathrm{X}+0,022 * \mathrm{X}^{2} \\
\mathrm{R}^{2} & =0,35\end{aligned}$ & $\begin{aligned} \hat{\mathrm{Y}} & =916,000+353,720 * * X \\
\mathrm{R}^{2} & =0,85\end{aligned}$ \\
\hline
\end{tabular}

* e** significativos a 5 e 1\% pel o teste $F$, respectivamente. Os val ores entre parênteses representam a significância dos coeficientes de regressão não significativas nem a $5 \%$.

conteúdo total da testemunha, os decréscimos atingiram 43\%, para $\mathrm{N}$; $57 \%$, para $\mathrm{P}$; $31 \%$, para K; $68 \%$, para $\mathrm{Ca}$; $68 \%$, para $\mathrm{Mg}$, e $50 \%$, para o $\mathrm{S}$, confirmandoqueP, Ca eMg foram os nutrientes mais afetados.

Esses resultados corroboram as afirmações de F oy (1974), segundo o qual o excesso deAl, além de inibir a formação normal das raízes, interfere nas reações enzimáticas, regula a disposição de polissacarídios nas paredes celulares e interfere na absorção, no transporte e no uso de vários nutrientes, inclusive deP, Ca eMg.

Considerando as duas maiores doses do el emento, verificou-se que o teor de fósforo na raiz foi maior do que o contido na parte aérea (Figura 2). A redistribuição deP em plantas estressadas por al umínio revelou acúmulo nas raízes e diminuição em órgãos da parte aérea (Furlani, 1989). I sso deveu-se à reação de contínua adsorção- preci pitação que reduz a entrada do nutriente nas raízes e, conseqüentemente, reduz seu transporte na parte aérea (Clarkson 1969). Quanto ao Ca, uma das prováveis hipóteses de sua redução (Figura 2) sustenta-se nas afirmações de Siegel \& Haug (1983), que relataram a interferência do Al na atividade da enzima ATPase estimulada pela calmodulina (Cam), uma proteína que exerce papel importante na manutenção de membranas e que constitui o principal alvo do alumínio.

Segundo Cambraia (1989), a calmodulina se faz presente na forma de um polipeptídeo com quatro sítios de ligação para cálcio, o qual pode agir como segundo mensageiro de um sistema regulatório de vários processos metaból icos importantes. Ainda de acordo com o autor, o cálcio, ao se ligar à Cam, provoca mudanças de conformação nesta proteína, alterando sua interação com certas enzimas e proteínas, tais como: fosfolipases, cinase do NAD, adenilciclase e $\mathrm{Ca}^{2+}$-ATPase. Desta maneira, é 

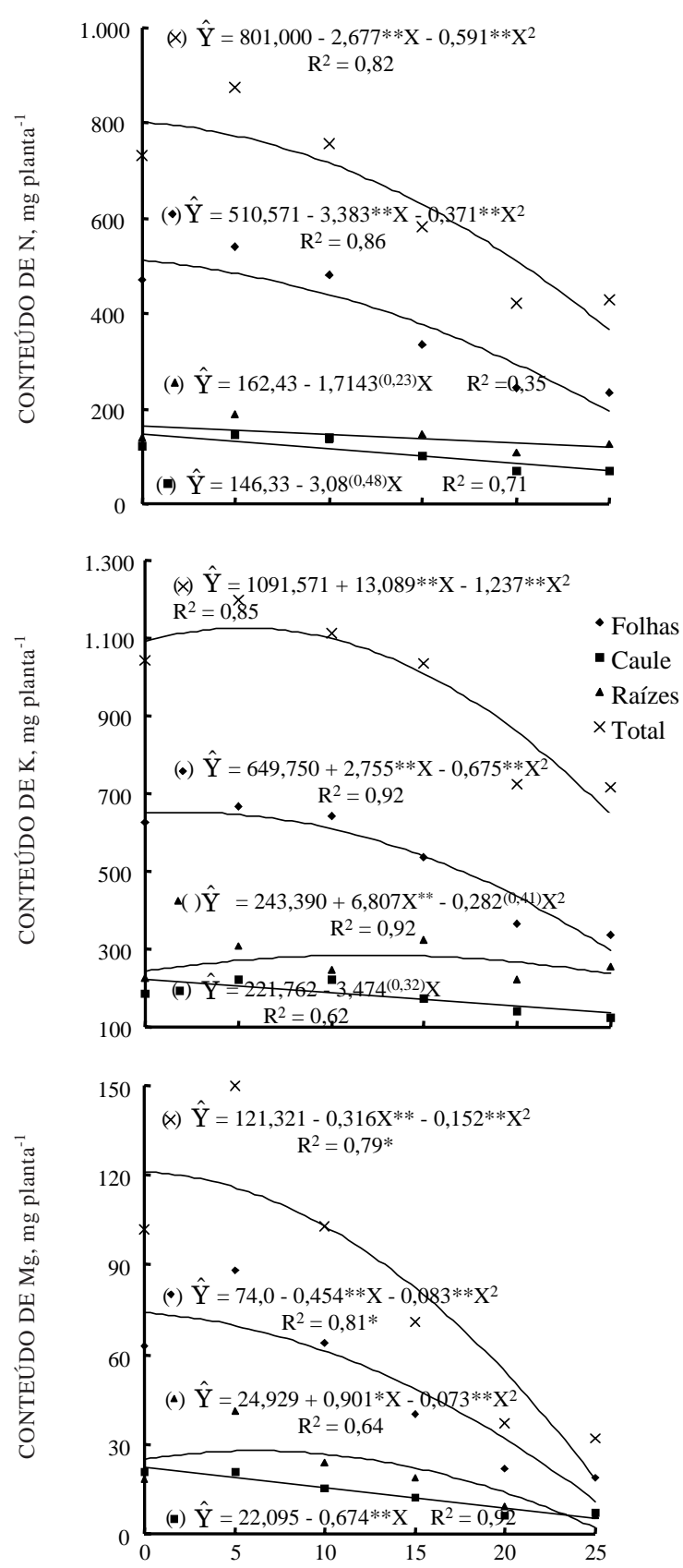
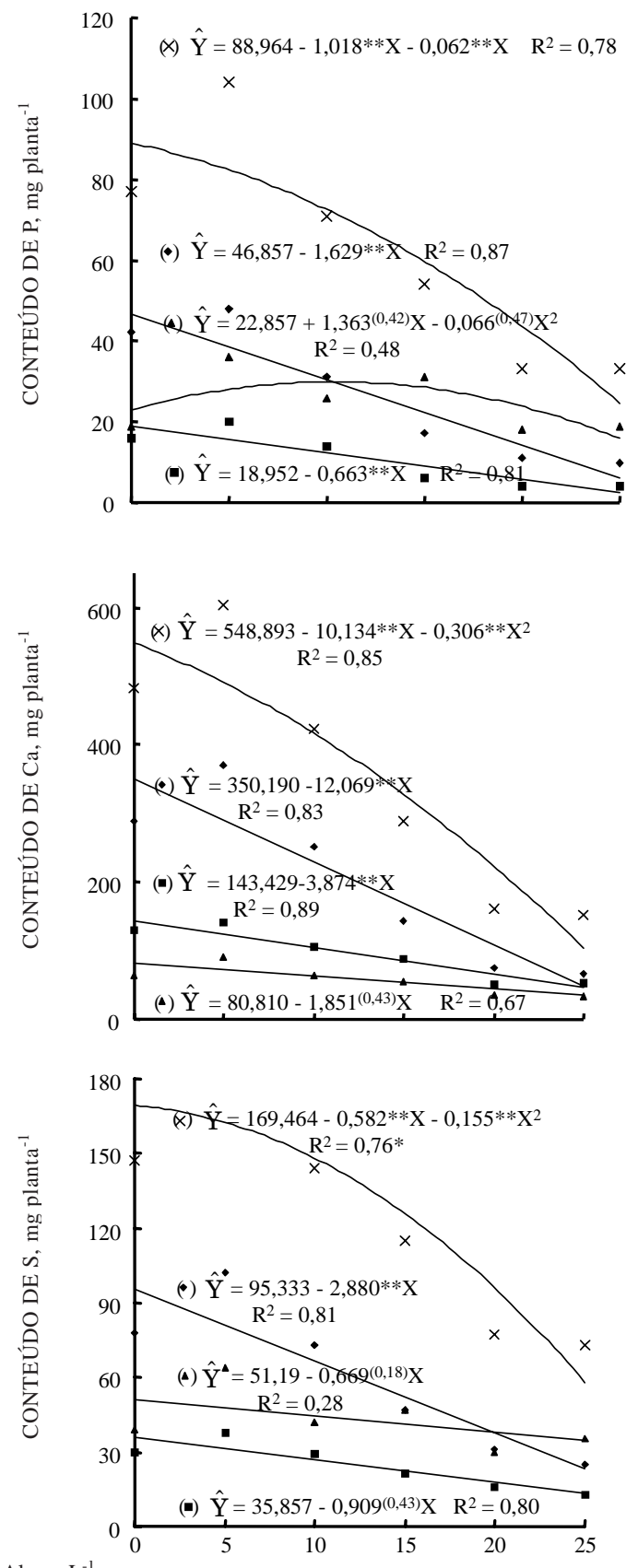

Figura 2. Conteúdo de macronutrientes nas fol has, caule, raízes e total nas mudas de goiabeira de acordo com as doses de alumínio (* e** significativos a 5 e $1 \%$ e os valores entre parênteses representam a significância dos coeficientes de regressão não significativos nem a 5\%).

possível que um dos efeitos fitotóxicos do Al esteja relacionado com a menor absorção de Ca, devido ao efeito de ini bição da proteína (Siegel \& Haug, 1983).

\section{Efeitos do alumínio na acumulação de micronu- trientes de alumínio nas folhas, caule e raízes}

As acumulações deCu, Fe, Mn, Zn eAl nas fol has, caule raízes mostraram reduções significativas nos tratamentos de maior adição dealumínio (Figura 3). Esses decréscimos foram, maiores notadamente, para Mn (69\%) e Zn (56\%). Para o Cu, as reduções do conteúdo na parte aérea determinaram, em termos percentuais, aumentos nas raízes, em comparação com outras partes da planta (fol has e caule). Em relação aos demais nutrientes, os tratamentos influendaram significativamenteas proporções acumuladas estabel ecidas e entre as partes vegetais, mostrando que, em qualquer circunstância, o teor de Fe na raiz representou, em média, cerca de $70 \%$ em relação ao da parteaérea, e Mn eZn, 27 e 44\%, respectivamente. 

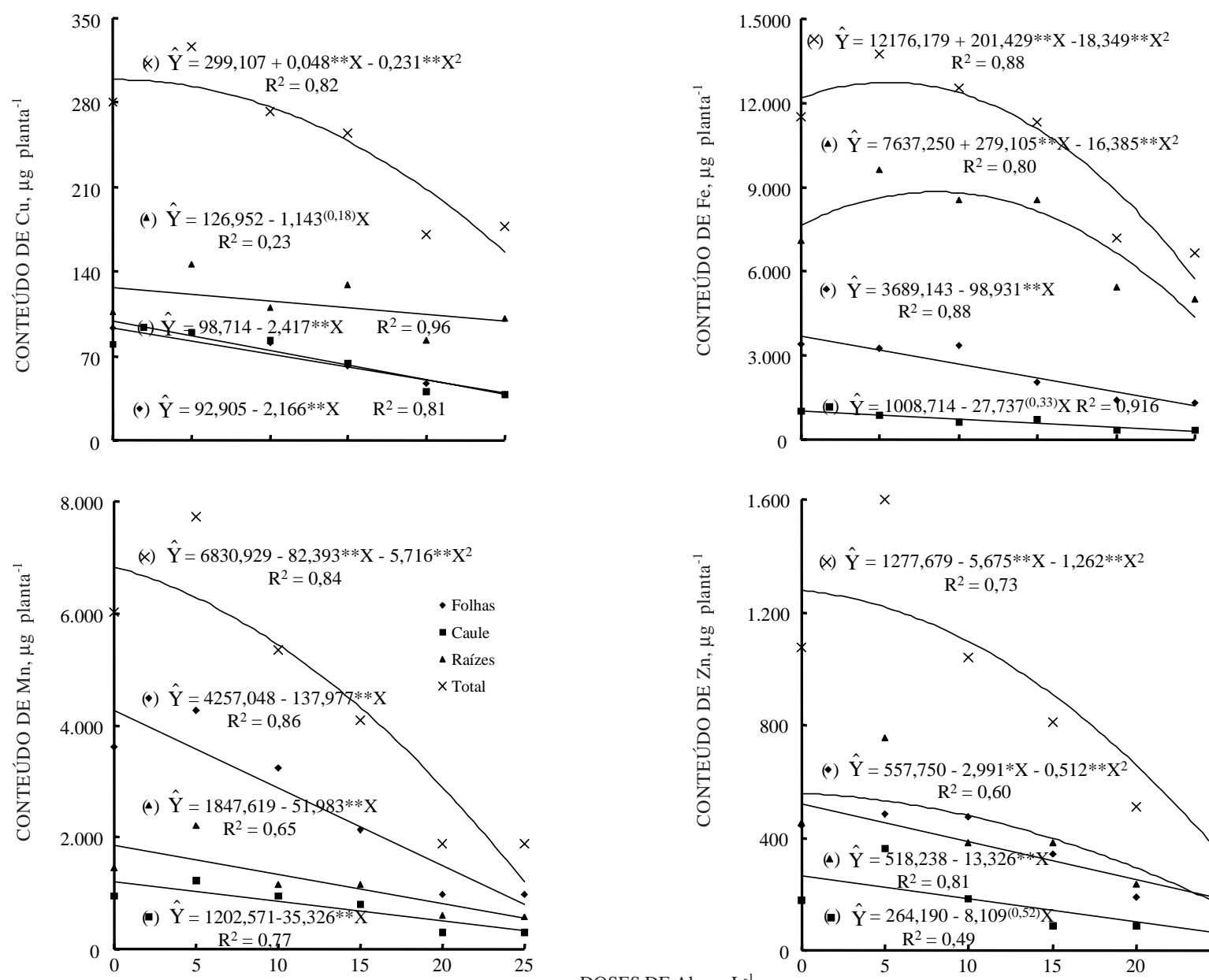

DOSES DE Al, mg L-1
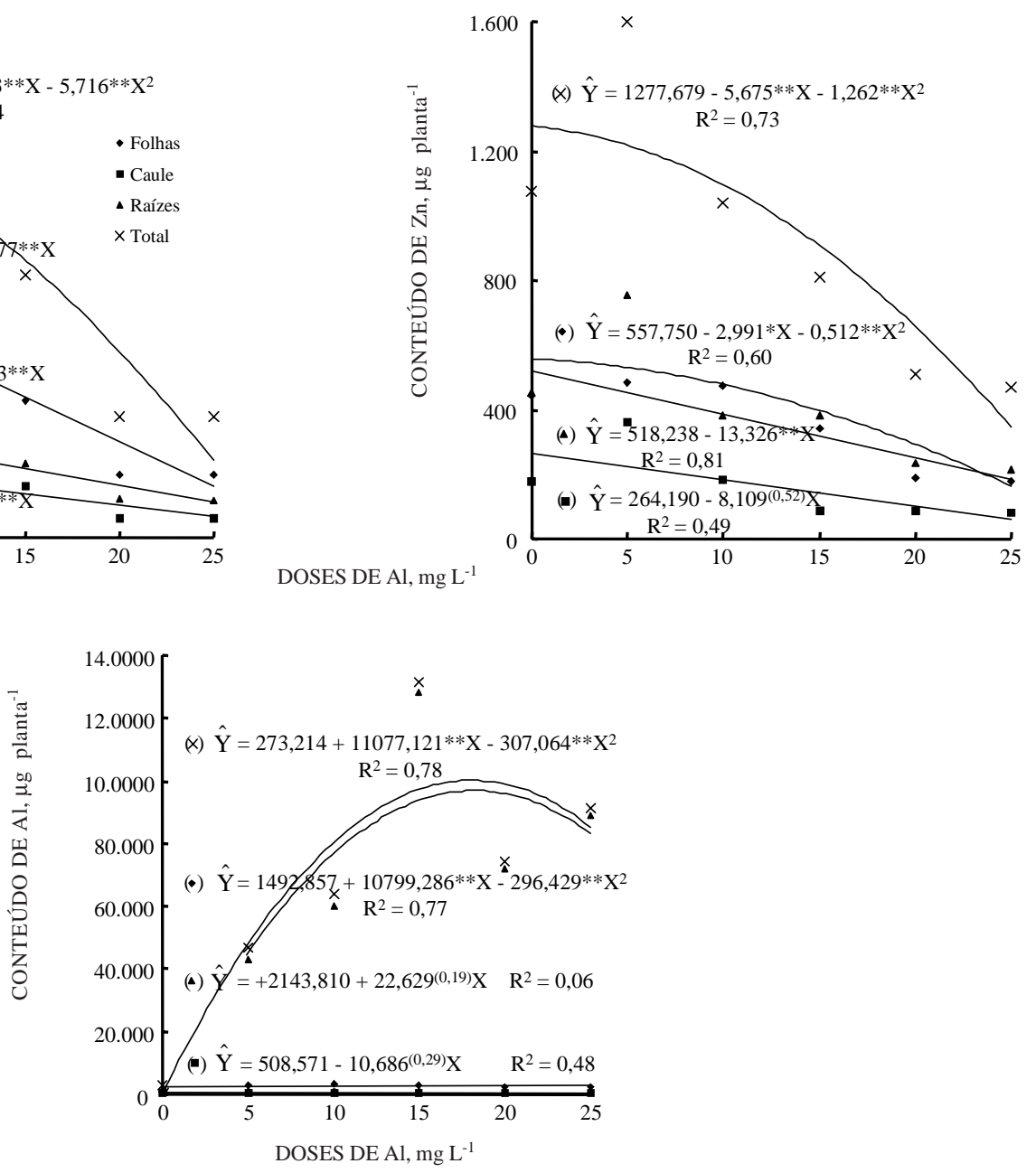

Figura 3. Teores de micronutrientes e alumínio nas folhas, caule, raízes e total nas mudas de goiabei ra de acordo com com as doses de alumínio (* e ** significativos a 5 e $1 \%$ e os valores entre parênteses representam a significância dos coeficientes de regressão não significativos nem a 5\%).

As adições de Al conferiram aumentos significativos no seu conteúdo total (Figura 3). As plantas submetidas à dose de $5 \mathrm{mg} \mathrm{L}^{-1}$ de Al apresentaram 17 vezes mais al umínio acumulado do que as da testemunha, com índices atingindo
30 vezes nas doses mai ores. Em todos os tratamentos em que ele foi adicionado, seu acúmulo nas fol has eqüivaleu-se cerca de três vezes àquele obtido no tratamento-testemunha. O conteúdo do caule, no entanto, não foi influenciado significativamente 
pelos tratamentos. A raiz foi o componente vegetal de maior conteúdo de Al (Figura 3), como conseqüência de sua adição, sendo diretamente proporcional aos teores encontrados nesta parte vegetal (Quadro 3). Com base no cál culo da relação parte aérea/raiz, obteve-se um quociente de índice próximo a 1 no tratamento testemunha (proporções equivalentes dealumínio entreas partes), enquanto, nas maiores concentrações de alumínio, esse quociente foi de 0,03 .

\section{CONCLUSÃO}

1. Em solução nutritiva, as mudas de goiabeira beneficiaram-se de concentrações de até $10 \mathrm{mg} \mathrm{L}$-1 de alumínio.

2. A maior concentração de Al reduziu a absorção deP, Ca, Mg, S, FeeMn, afetando otransportedesses nutrientes para a parte aérea.

3. A redução dos teores de $\mathrm{Ca}$ e $\mathrm{Mg}$ deveu-se, provavelmente, a uma inibição interiônica desses cátions pelo Al.

4. A goiabeira, na sua faseinicial, nãofoi tolerante ao alumínio nas concentrações acima de 10 mg L-1.

\section{LITERATURA CITADA}

ASHER, C.J . Beneficial elements, functional nutrients, and possiblenew essential elements. In: MORTVEDT, J . . ; COX, F.R.; SHUMAN, L.M. \& WELCH, R.M., eds. Micronutrients in Agriculture. Madison, American Society of America, 1991. p.703-723.

BRACCINI, M.C.L.; MARTINEZ, H.E.P.; PEREIRA, P.R.G.; SAMPAIO, N.F. \& SILVA, E.A.M. Comportamento de populações de café quanto a tolerância ao alumínio em solução nutritiva. II - Efeito sobre a composição química e eficiência nutricional das plantas. In: CONGRESSO BRASILEIRO DE CIÊNCIA DO SOLO, 25., Viçosa, 1995. Resumos expandidos. Viçosa, Sociedade Brasileira de Ciência do Solo, 1995. p.1360-1361.

BUENO N. Alguns aspectos recentes da nutrição mineral do cupuaçuzeiro. In: SEMINÁRIO INTERNACIONAL SOBRE PIMENTA-DO-REINO E CUPUAÇU, 1., Belém, 1997. Anais. Belém, EMBRAPA Amazônia Oriental/IICA, 1997. p.79-80.

CAMARGO, O.A. \& FURLANI, P.R. Alumínio no solo: concentração, especiação e efeito no desenvolvimento radicular. In: SIMPÓSIO AVANÇADO DE SOLOS E NUTRIÇÃO DE PLANTAS, 2., Piracicaba, 1989. Anais. Campinas, Fundação Cargill, 1989. p.45-69.

CAMBRAIA, J . Mecanismos de tolerância a toxidez de alumínio em plantas. In: REUNIÃO BRASILEIRA DE FISIOLOGIA VEGETAL, 2., Piracicaba, 1989. Anais. Piracicaba, Sociedade Brasileira de Fisiologia Vegetal, 1989. p.85-92.
CLARKSON, D.T. Metabolic aspects of aluminum toxicity and some possible mechanisms for resistance. In: ROBINSON, I.H., ed. Ecological aspects of mineral nutrition of plants. Oxford, Blackwell Scientific, 1969. p.381-397.

FAGERIA, N.K. \& ZIMMERMANN, F.J .P. Seleção de cultivares de arroz para tolerância à toxidez de alumínio em solução nutritiva. Pesq. Agropec. Bras., 14:41-47, 1979.

FAQUIN, V. \& VALE, F.R. Toxidez dealumínio e manganês. I nf. Agropec., 15:28-38, 1991.

FOY, C.D. Effect of aluminum on plant growth. In: CARLSON, F.W., ed. The plant root and its environment. Charlottesville, University Press of Virginia, 1974. p.601-640.

FOY, C.D.; CHANEY, R.L. \& WHITE, M.C. The physiology of metal toxicity in plants. Ann. Rev. Plant Physiol., 29:511566, 1978.

FOY, C.D. Physiological effects of hydrogen, aluminum and manganese toxicities in acid soil. In: ADAMS, F., ed. Soil acidity and liming. Madison, American Society of Agronomy, 1984. p.57-97.

FOY, C.D. Soil chemical factors limiting plant root growth. In: HATFIELD, J .L., ed. Limitations to plant root growth. New York, Springer-Verlag, 1992. p.97-149.

FURLANI, P.R. Toxicidade de alumínio e manganês em plantas. In: RAIJ , B. van; BATAGLIA, O.C. \& SILVA, N.M. Acideze calagem no Brasil. Campinas, Sociedade Brasileira de Ciência do Solo, 1983. p.79-85.

FURLANI, P.R. Efeitos fisiológicos do alumínio em plantas. In: SIMPÓSIO AVANÇADO DE SOLOS E NUTRIÇÃO DE PLANTAS, 2., Piracicaba, 1989. Anais. Campinas, Fundação Cargill, 1989. p.73-90.

GONZAGA NETO L. \& SOARES J .M. A cultura da goiabeira. Brasília, Empresa Brasileira de Pesquisa Agropecuária, 1995. $75 p$.

HOAGLAND, D.R. \& ARNON, D.I. The water culture method for growing plants without soils. Berkeley, California Agric. Exp. St., 1950. 347p.

LOPES, A.S. Solos sob "Cerrado": características, propriedades e manejo. Piracicaba, POTAFOS, 1984. 162p.

MALAVOLTA, E. Práticas de nutrição mineral de plantas. Curso de Pós-Graduação em Solos e Nutrição de Plantas. Piracicaba, Escola Superior deAgricultura Luiz deQueiroz, 1976. 65p.

MALAVOLTA, E.; VITTI, G.C. \& OLIVEIRA, S.A. Avaliação do estado nutricional das plantas; princípios e aplicações. Piracicaba, POTAFOS, 1997. 319p.

MARSCHNER, H. Mineral nutrition of higher plants. London, Academic Press, 1995. 889p.

MARTINEZJ r., J .R. \& PEREIRA, F.M. Respostas da goiabeira a diferentes quantidades de N, P e K. In: CONGRESSO BRASILEIRO DE FRUTICULTURA, 8., Brasília, 1986. Anais. Brasília, Sociedade Brasileira de Fruticultura, 1986. p.293-296 
MULLETTE, K.J . Stimulations of growth in eucalyptus due to aluminum. Plant \& Soil, 42:495-599, 1975.

NATALE, W.; COUTINHO, E.L.M.; BOARETTO, A.E. \& PEREIRA F.M. Goiabeira; calagem eadubação. J aboticabal, FUNEP, 1996. 22p.

OLIVEIRA P.I \& MALAVOLTA E. Efeito do alumínio e do manganês no feijoeiro (Phaseolus vulgaris L.). Pesq. Agropec. Bras., 17:549-557, 1982.

OLIVEIRA, L.E.M. Crescimento e comportamento nutricional de cultivares de mandioca (Manihot esculenta, Grantz), submetidos a níveis de alumínio. Viçosa, Universidade Federal de Viçosa, 1979. 39p. (Tese de Mestrado)

PIMENTEL-GOMES, F. Curso de estatística experimental. Piracicaba, Nobel, 1990. 468p.
SALVADOR, J .O.; MOREIRA, A. \& MURAOKA, T. Deficiência nutricional em mudas de goiabeira (Psidium guajava L.) decorrente da omissão simultânea de dois macronutrientes. Pesq. Agropec. Bras., 33:1623-1631, 1998.

SALVADOR, J .O.; MOREIRA, A. \& MURAOKA, T. Efeito da omissão combinada de $\mathrm{N}, \mathrm{P}, \mathrm{K}$ e S nos teores foliares de macronutrientes degoiabeira. Sci. Agric., 56:501-507, 1999a.

SALVADOR, J .O.; MOREIRA, A. \& MURAOKA, T. Sintomas visuais de deficiências de micronutrientes e composição mineral de fol has em mudas de goiabeira. Pesq. Agropec. Bras., 34:1655-1662, 1999b.

SIEGEL, N. \& HAUG, A. Calmodulin - dependent formation of membrane potential in barley root plasma membrane vesicles: A biochemical model of aluminum toxicity in plants. Physiol. Plant., 59:285-291, 1983. 